\title{
A Patch Density Recommendation based on Convergence Studies for Vehicle Panel Vibration Response resulting from Excitation by a Diffuse Acoustic Field
}

\author{
Andrew Smith, ${ }^{1}$ \\ NASA/Marshall Space Flight Center, Huntsville, AL, 35812 \\ Bruce LaVerde ${ }^{2}$ \\ ERC, Huntsville, AL, 35812 \\ Douglas Jones ${ }^{3}$ Robert Towner ${ }^{4}$, \\ Jacobs Engineering, Huntsville, AL, 35812 \\ Ron Hunt ${ }^{5}$ \\ Bevilacqua Research Corporation, ESSSA Group, Huntsville. AL, 35612, USA
}

\begin{abstract}
Fluid structural interaction problems that estimate panel vibration from an applied pressure field excitation are quite dependent on the spatial correlation of the pressure field. There is a danger of either over estimating a low frequency response or under predicting broad band panel response in the more modally dense bands if the pressure field spatial correlation is not accounted for adequately. Even when the analyst elects to use a fitted function for the spatial correlation an error may be introduced if the choice of patch density is not fine enough to represent the more continuous spatial correlation function throughout the intended frequency range of interest. Both qualitative and quantitative illustrations evaluating the adequacy of different patch density assumptions to approximate the fitted spatial correlation function are provided. The actual response of a typical vehicle panel system is then evaluated in a convergence study where the patch density assumptions are varied over the same finite element model. The convergence study results are presented illustrating the impact resulting from a poor choice of patch density. The fitted correlation function used in this study represents a Diffuse Acoustic Field (DAF) excitation of the panel to produce vibration response..
\end{abstract}

\section{Nomenclature}

$\mathrm{DAF}=$ Diffuse Acoustic Field

eq $=$ Equation

$\mathrm{FEM}=$ Finite Element Model

MSFC $=$ Marshall Space Flight Center

NESC $=$ NASA Engineering and Safety Center

$\mathrm{SPL}=$ Sound Pressure Level

1 Vibroacoustics Specialist, MSFC/EV31, AIAA Member.

2 Vibroacoustics Lead Engineer, Support to MSFC/EV31, AIAA Member.

3 Structural Dynamics \& Loads Specialist, Jacobs Engineering, 1500 Perimeter Parkway, Huntsville, AL, AIAA Member.

4 Structural Dynamics \& Loads Specialist, Jacobs Engineering, 1500 Perimeter Parkway, Huntsville, AL, AIAA Member.

5 Structural Dynamics Analyst, Bevilacqua Research Corporation, Supporting MSFC/ES22, Huntsville, AL. 


\section{Introduction}

$\mathrm{T}$ HE importance of adequately representing the pressure field forcing function used in structural response studies relying over the surface of a structural finite element model is illustrated in the following sections of the paper (Figure 1). The response resulting from acoustic noise excitation calculated using such models is an example of a fluid structural interaction that depends on the spatial correlation of the applied pressures at each frequency of interest. The patch method can be used to approximate a continuously changing spatial correlation function by sampling the values of that function at regular intervals and applying them consistently within each patch. This approximation approaches the continuous function in the limit as the size of each patch gets smaller and the patch density gets larger. Since choosing an extremely fine patch density can be computationally expensive, the recommendations of the technical paper are intended to assist the analyst in avoiding errors, and to provide suitably
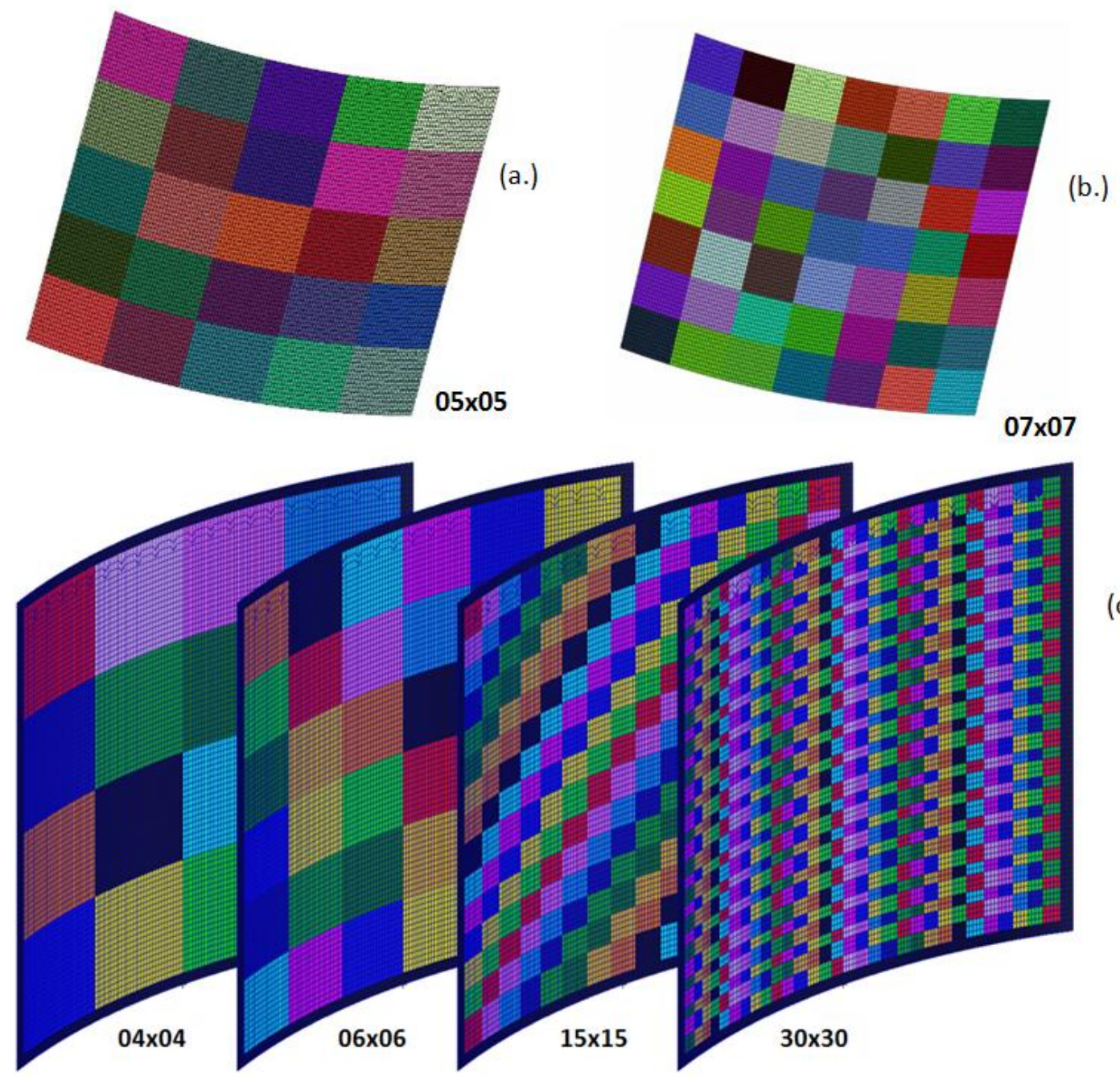

(c.)

Figure 1. Patches are defined on the surface of a vehicle panel Finite Element Model in order to specify spatial cross correlation relationships for the applied forcing function. (a.) a05x05 Patch density with patch center to center distance of $16.2 \mathrm{in}$. (b.) a $07 \times 07$ patch density with adjacent patch center to center distance of 11.6 in. (c.) a range of other possible patch density assumptions depicted over the same panel.

accurate response results for the frequency bands important to their own work.

Using the patch method to represent the continuous spatial correlation function of a phased pressure field over a structural surface is an approximation. The approximation approaches the continuous function as the patch size becomes smaller. 
As a first step, several comparisons of the approximation versus the continuous function realized at different frequencies are provided. These plots may provide insight revealing the answers to the following questions:

1. For what patch size/density does the approximation seem to be very good?

2. What does the comparison look like when the patch size is too coarse (i.e. too large) for the frequency range and the approximation begins to break down?

3. What does the approximation look like when the patch size is grossly too large?
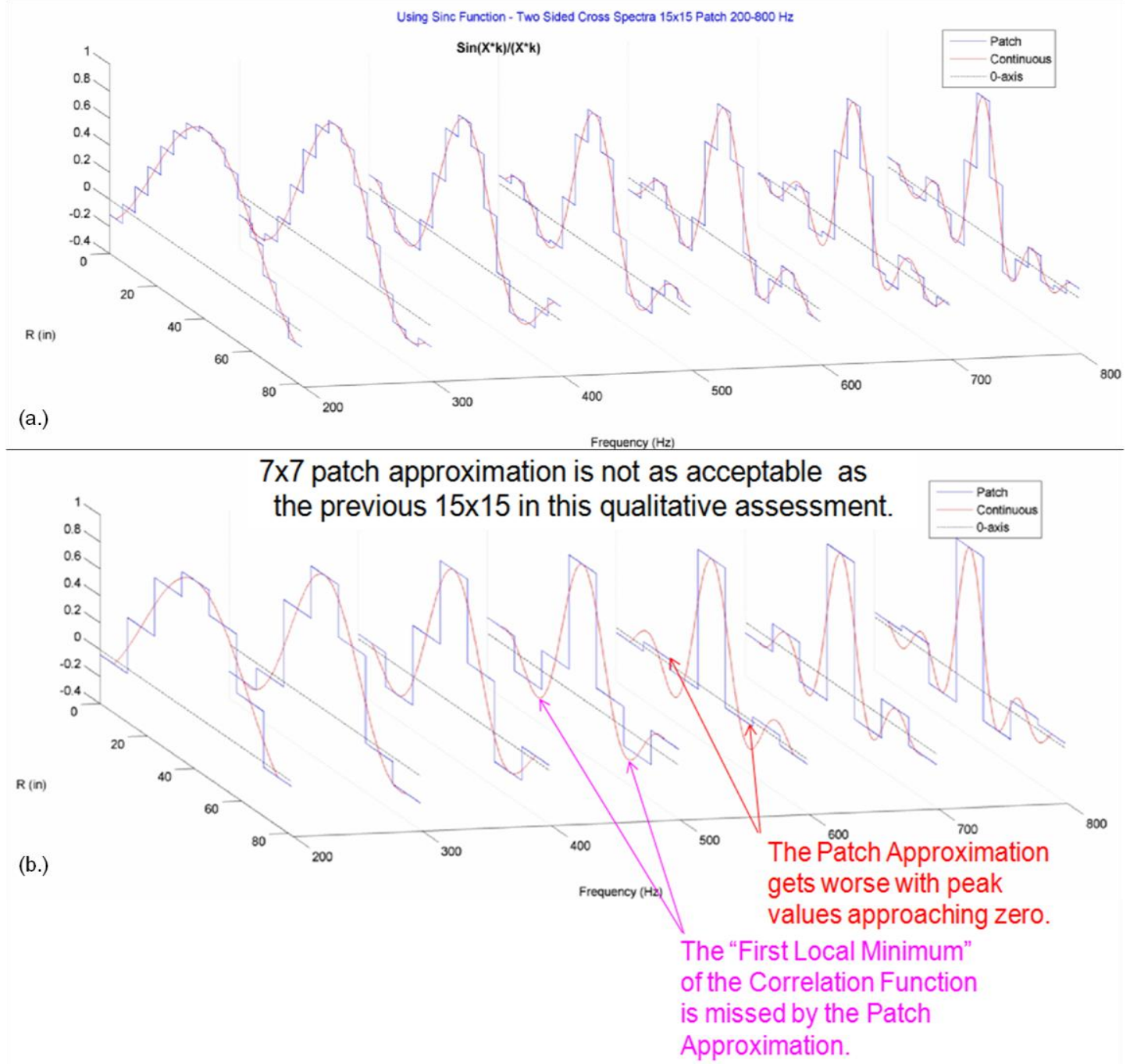

Figure 2. Waterfall plots depicting the approximation of a continuous spatial function that depends also on frequency. (a.) Consider the 15x15 Patch density Assumption evaluated in one dimension across frequency. (b.). The approximation of the continuous function is not as good for the $07 \times 07$ patch density evaluated the same way.

These observations can be normalized with respect to frequency by converting the patch dimensions so that they represent a fraction of the fluid wave length using the speed of sound (Figure 3). Imposing the applied pressure field that represents the correlation with local maxima and minima across the surfaces of the panel is desired. Therefore, care must be taken to avoid an approach that flattens out the local maxima and minima to zero. Figure 3a indicates that choosing a patch center to center distance equal to the $1 / 2$ the fluid wave length would result in this undesired situation. Therefore, selecting a center to center distance equal to $1 / 2$ of the wavelength at the largest frequency of interest would be too coarse an assumption for patch density. The assumed patch density should be finer. Patch 


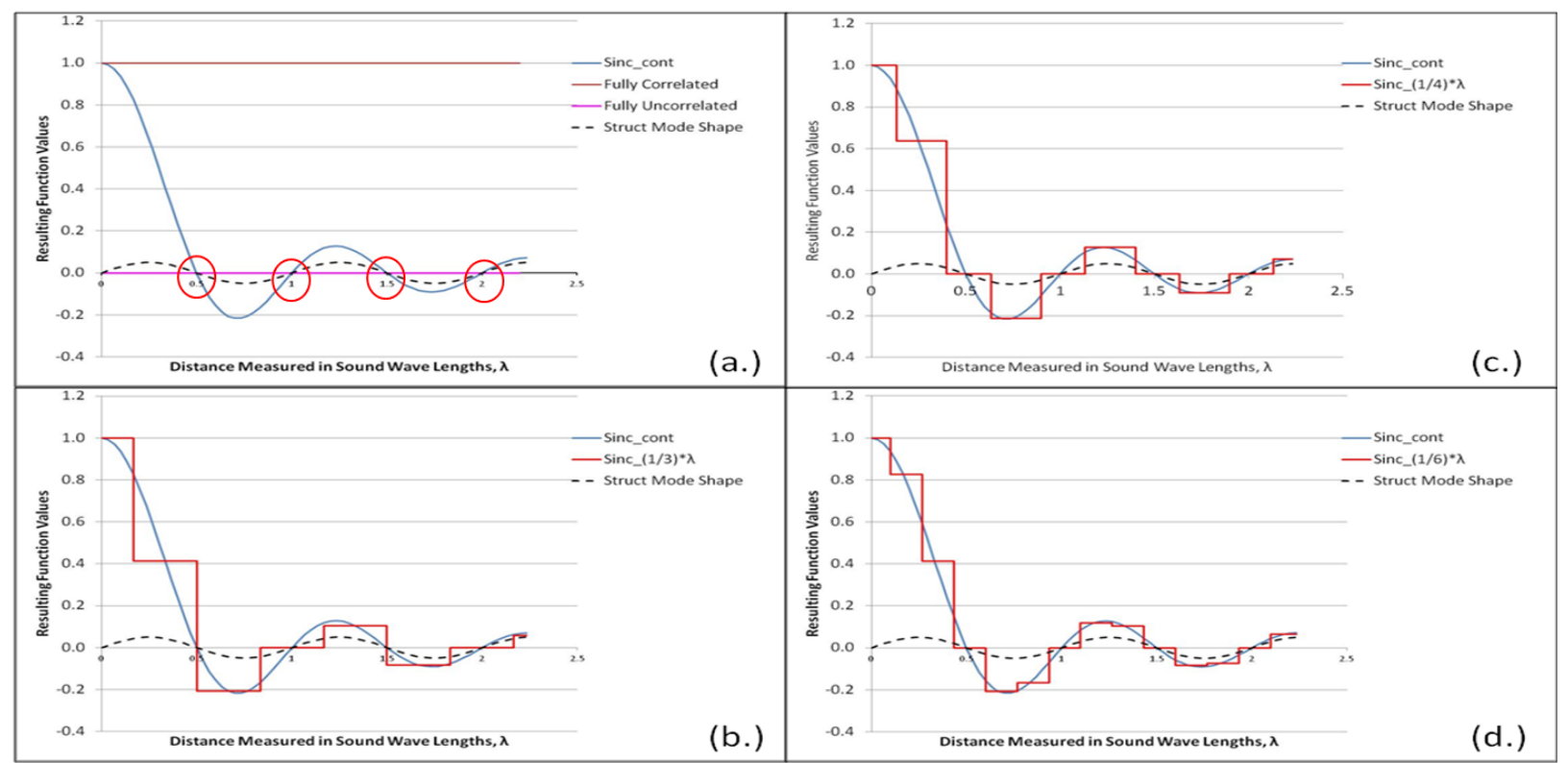

Figure 3. Spatial Correlation Function Normalized to Fluid Wavelength (a.) DAF Sinc Correlation Function compared to a proposed Structural Panel Bending Mode Shape. Note that zeros occur at half wavelengths. (b.). Approximation Assuming (1/3) $\lambda$ Patch Size. (c.). Approximation Assuming (1/4) $\lambda$ Patch Size. (d.). Approximation Assuming (1/6)ג Patch Size.

densities corresponding to $1 / 3$ or $1 / 4$ of the fluid wavelength at the highest frequency of interest should be evaluated for adequacy. II.

An explanation describing how the correlation function is used to apply the pressure field is discussed in Section

In addition to the qualitative comparisons provided above, the enclosed area of the correlation function is explored visually in Section III. This provides a more quantitative assessment of the spatial distribution of phased pressures across the panel surface.

Afterward, a convergence study is presented using a single launch vehicle panel FEM to demonstrate by comparison the impact of using a poor patch density assumption to complete the analysis. Developing insights that help us to predict sufficient patch density to provide adequate convergence within the intended frequency range of interest for our proposed analyses is a goal of this study.

\section{The Applied Pressure Forcing Function depends on the Spatial Correlation Function}

The equations presented below provide the framework for applying a pressure field excitation over the surface of a panel system. A random pressure field on a launch vehicle skin surface may be approximated by dividing the surface into patches, or regions of uniform pressure with no phase offsets within the patch. The size of each patch must be chosen to be small enough to justify the assumption of uniform (though dynamically varying) pressure with zero phase offset across the patch. The selection of the appropriate patch and element size is an important parameter for specifying the forcing function. The pressure may be defined as a stationary Gaussian random field with spatially varying autospectral density. The pressures on any pair of patches may be correlated, exhibiting a non-zero cross-spectral density between them. The random pressure field is thus a Hermitian matrix of spectral densities of dimension $\mathrm{Np}$, the total number of pressure patches. The pressure autospectra occur on the diagonal of the matrix. The cross-spectra appear as off-diagonal terms. The random pressure field on all patches may be written as 


$$
\boldsymbol{P}_{N_{p}}(\omega)=\left[\begin{array}{cccc}
P_{11} & P_{12} & \cdots & P_{1 N_{p}} \\
P_{21} & P_{22} & \cdots & P_{2 N_{p}} \\
\vdots & \vdots & \ddots & \vdots \\
P_{N_{p} 1} & P_{N_{p} 2} & \cdots & P_{N_{p} N_{p}}
\end{array}\right]
$$

where $P_{b c}=P_{c b}^{*}$, and the asterisk denotes the conjugate operator. If spatial functions $\gamma(\omega, R)$ are defined that relate the autospectra to the cross-spectra, eq 1 may be written as

$$
\boldsymbol{P}_{N_{p}}(\omega)=\left[\begin{array}{cccc}
\gamma_{11} \hat{P}_{11} & \gamma_{12} \hat{P}_{12} & \cdots & \gamma_{1 N_{p}} \hat{P}_{1 N_{p}} \\
\gamma_{21} \hat{P}_{12} & \gamma_{22} \hat{P}_{22} & \cdots & \gamma_{2 N_{p}} \hat{P}_{2 N_{p}} \\
\vdots & \vdots & \ddots & \vdots \\
\gamma_{N_{p} 1} \hat{P}_{1 N_{p}} & \gamma_{N_{p} 2} \hat{P}_{2 N_{p}} & \cdots & \gamma_{N_{p} N_{p}} \hat{P}_{N_{p} N_{p}}
\end{array}\right]
$$

where $\hat{P}_{b c}=\sqrt{P_{b b} P_{c c}}$ and $\gamma_{b b}$ have been added to the diagonals for generalization. The expression for $\hat{P}_{b c}$ arises from an inequality requirement on the coherence, which states that

$$
0 \leq \frac{\left|P_{b c}(\omega)\right|^{2}}{P_{b b}(\omega) P_{c c}(\omega)} \leq 1.0
$$

For a diffuse field, the spatial functions $\gamma$ may be expressed as

$$
\gamma_{b c}\left(\omega, R_{b c}\right)=\frac{\sin \left(R_{b c} \kappa(\omega)\right)}{R_{b c} \kappa(\omega)}
$$

where $R_{b c}$ is the distance between the centroids of patches $b$ and $c, \kappa(\omega)=\omega / C_{o}$, and $C_{o}$ is the speed of sound through the fluid medium adjacent to the panel/patch. The patch centroids are constrained to lie on the curved skin surfaces. When $b=c$, the spatial functions coincide with the patch autospectra, the distance $R$ between patches vanishes and $\gamma_{b b} \rightarrow 1.0$ in the limit as $R_{b b} \rightarrow 0$ (L'Hopital's Rule).

The relationship between patches has therefore been described in equation 4 and applied on the off diagonal terms of the matrix in equation 2. The expression in equation 4 was developed by Rafaely ${ }^{6}$. Approximation of the function represented by equation 4 is explored in the next section.

\footnotetext{
${ }^{6}$ (Rafaely, June 2000)
} 


\section{Quick Look Evaluation For Adequacy of the Approximation}

Short of performing a convergence study, an analyst might compare the look of the approximation provided by his choice of patch density to the function. They might also quantitatively assess the values of the approximation in regions where the function returns positive and negative values. Three types of areas were calculated in order to assess the values of the function in certain regions. These three area types are illustrated in figures 4 through 6 .

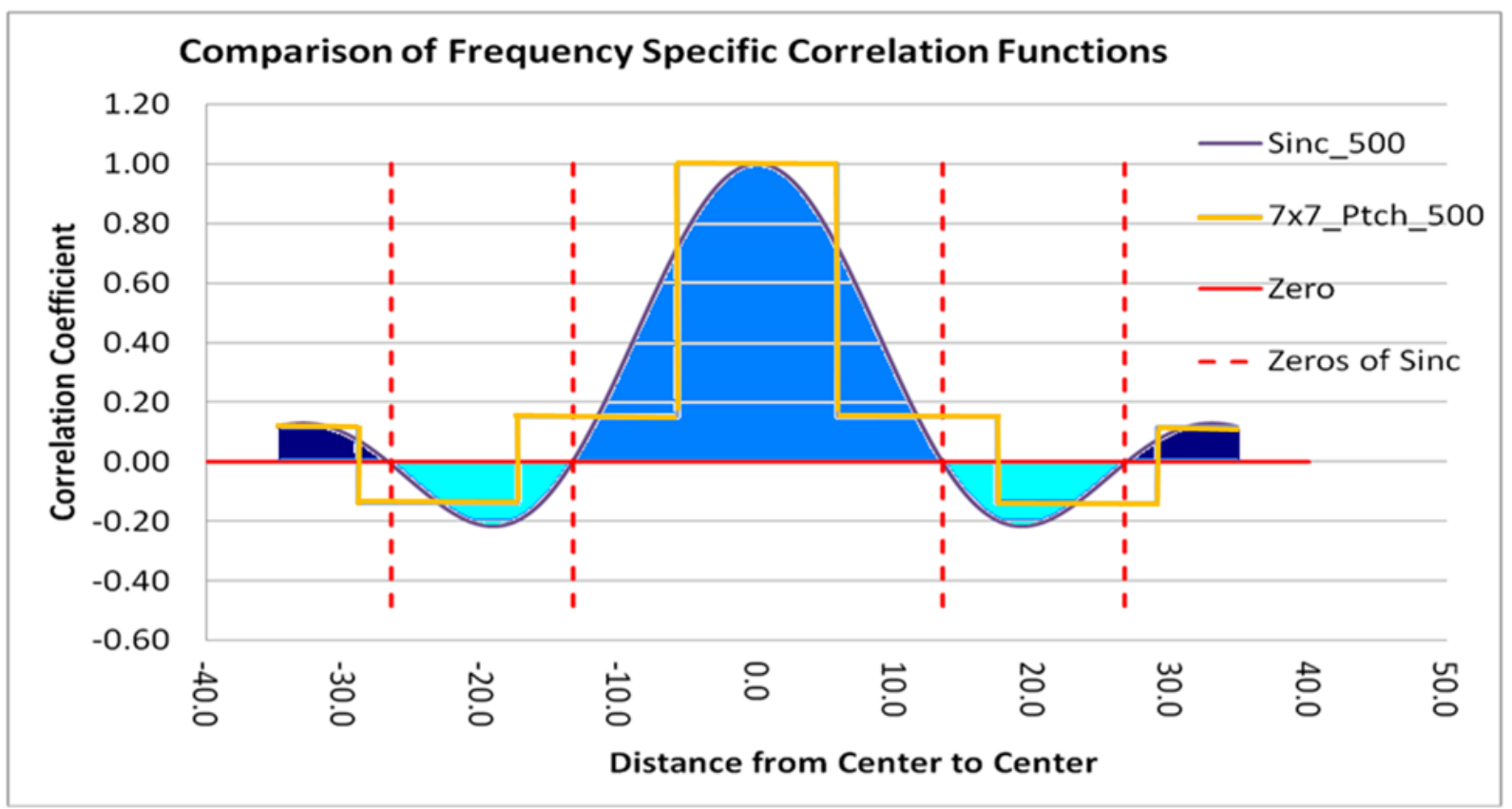

Figure 4. Spatial Correlation Function (i.e. the Sinc Function) Evaluated at $500 \mathrm{~Hz}$. Patch approximation of the same function at $500 \mathrm{~Hz}$. Enclosed area of the continuous Sinc correlation function is presented in shaded lobes above and below zero. This $500 \mathrm{~Hz}$ example shows that a $7 \times 7$ patch approximation is not ideal or adequate at this frequency. 


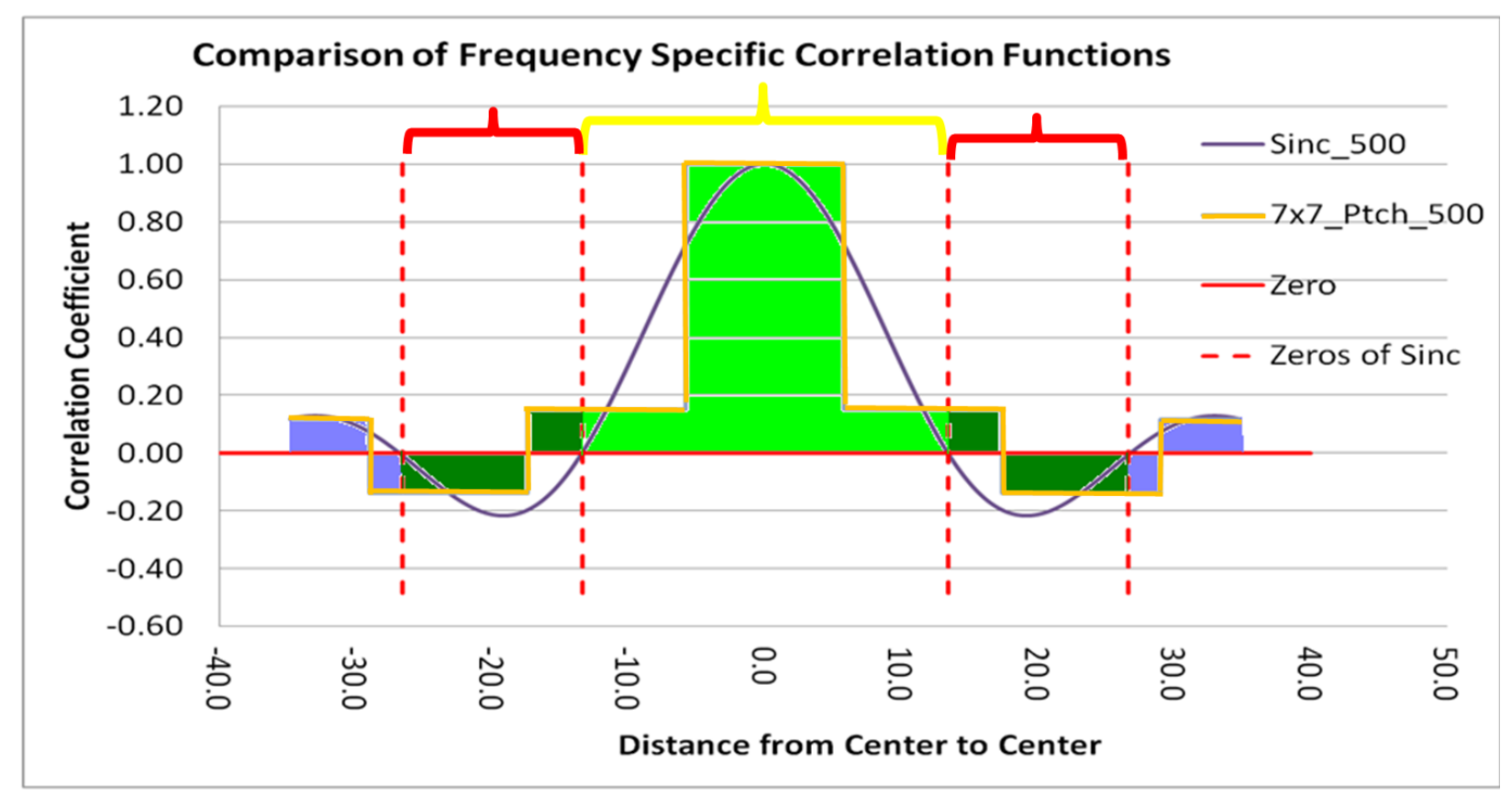

Figure 5. Spatial Correlation Function - Between the yellow bracketed Zeros the Function should be positive with positive enclosed area. Between the red bracketed Zeros the Function should be negative with negative enclosed area. The approximation is not perfect by these evaluation criteria. The net enclosed area in red is negative (but smaller negative).

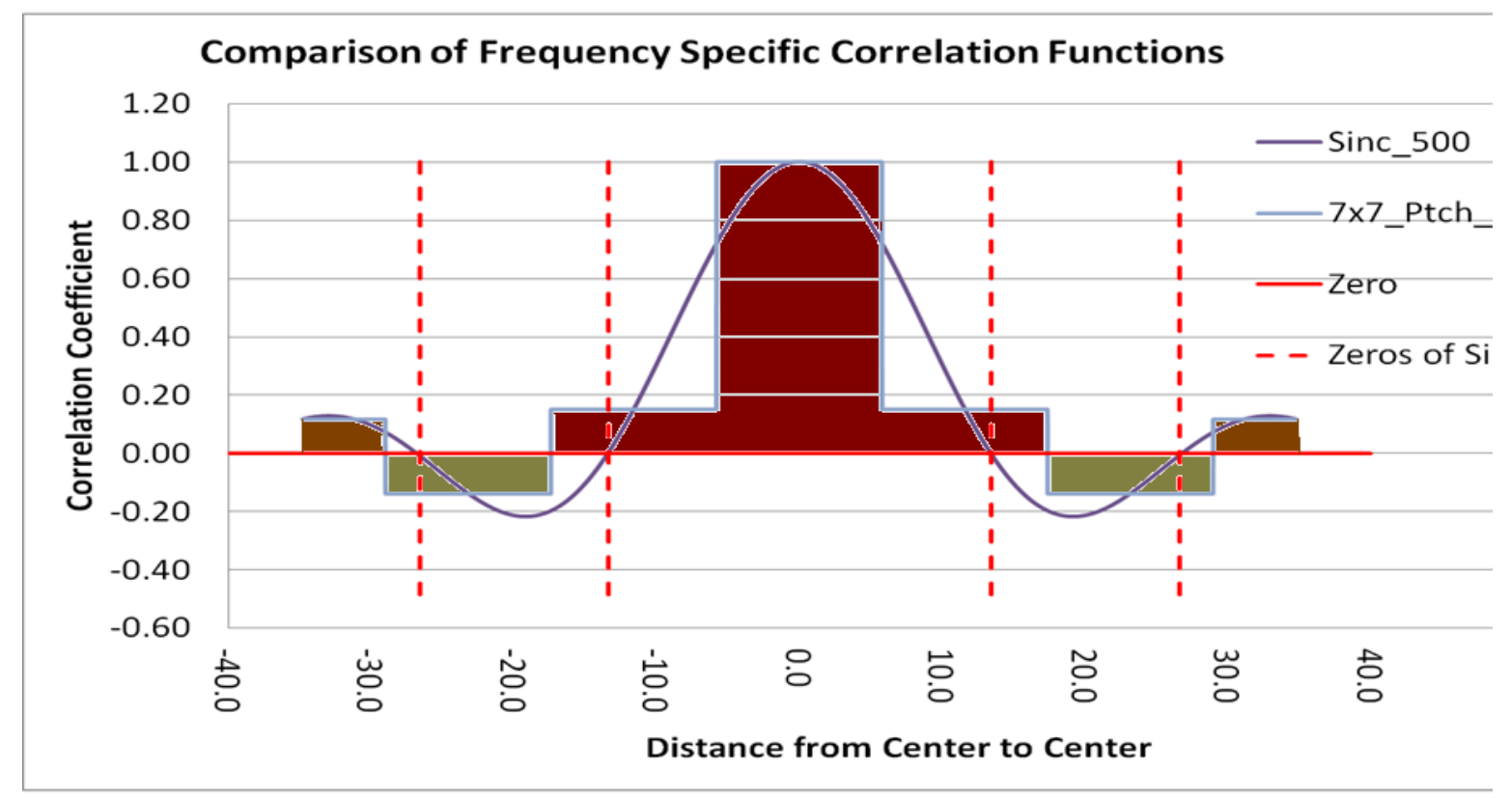

Figure 6. Spatial Correlation Function - The actual enclosed area of the $7 \times 7$ approximation is spatially distributed differently than the Sinc. The size of its realized positive and negative lobes was also evaluated without regard to the zeros of the Sinc function.

Since the $15 \times 15$ assessment appeared to be the better assumption of the two cases that were compared in Figure 2, Figures 7 though 12 are provided to show the differences of the applied approximation using comparisons with 
the continuous correlation function. First the shape and then the calculated areas are compared in different frequency ranges.

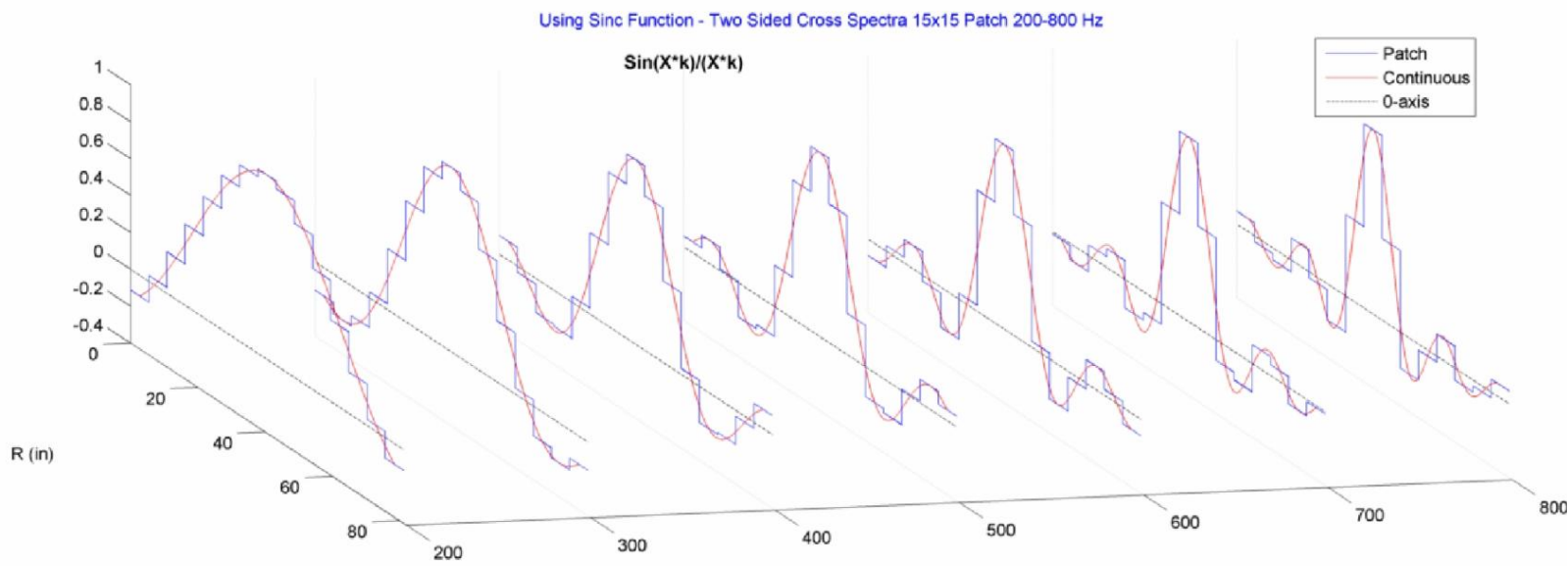

Figure 7. Spatial Correlation Function - The plot presents a single row of 15 patches from a $15 \times 15$ approximation of the Sinc spatial correlation function providing good approximations in the range from 200 to $800 \mathrm{~Hz}$.

The Sinc function is a function of wave-number and thus changes with frequency. A smaller patch size/higher patch density is required to accurately represent results at higher frequencies. The choice of patch density should depend on the highest frequency of interest for the structural response analysis.
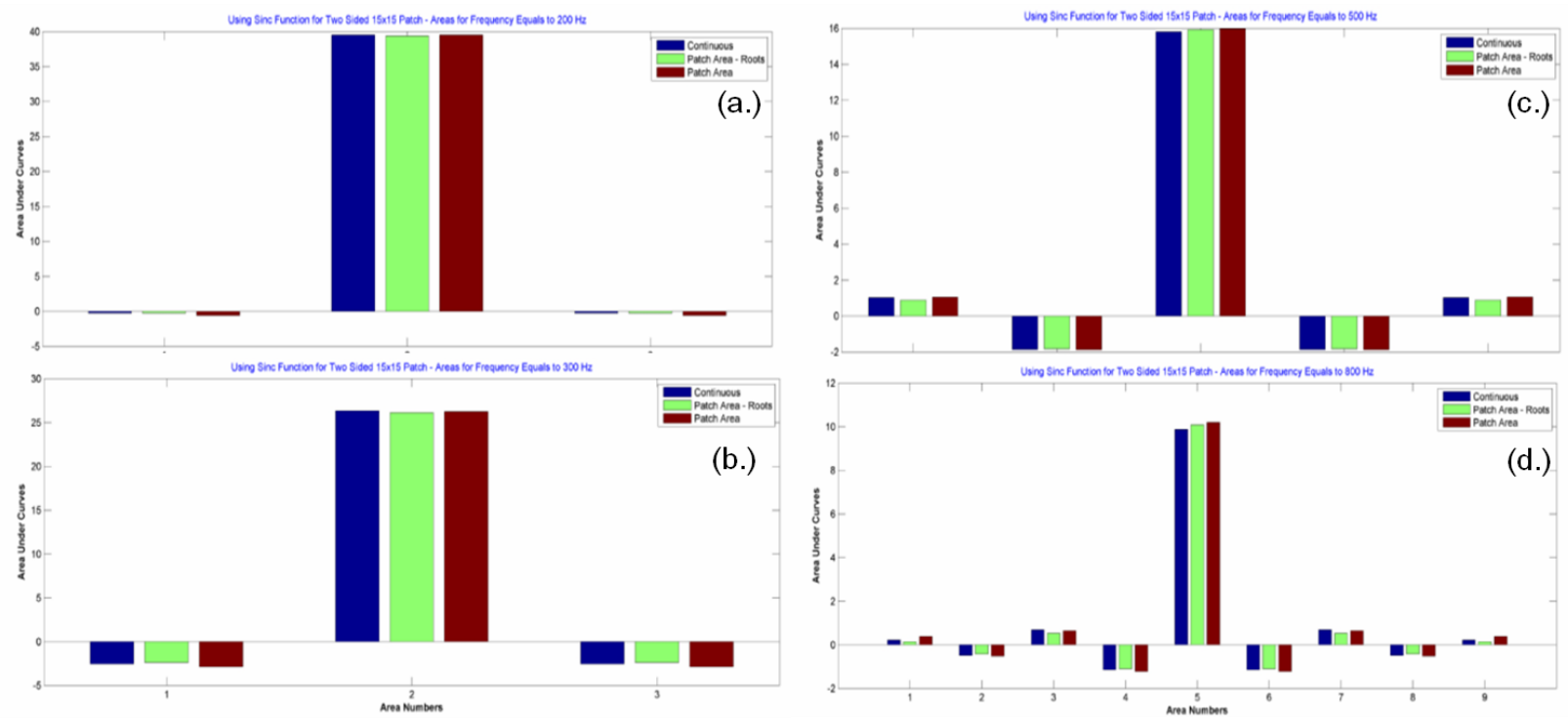

Figure 8. Spatial Correlation Function Area Comparisons: (a.) Correlation Area Comparison at $200 \mathrm{~Hz}$. (b.) Correlation Area Comparison at 300 Hz. (c.) Correlation Area Comparison at 500 Hz. (d.) Correlation Area Comparison at $800 \mathrm{~Hz}$. 


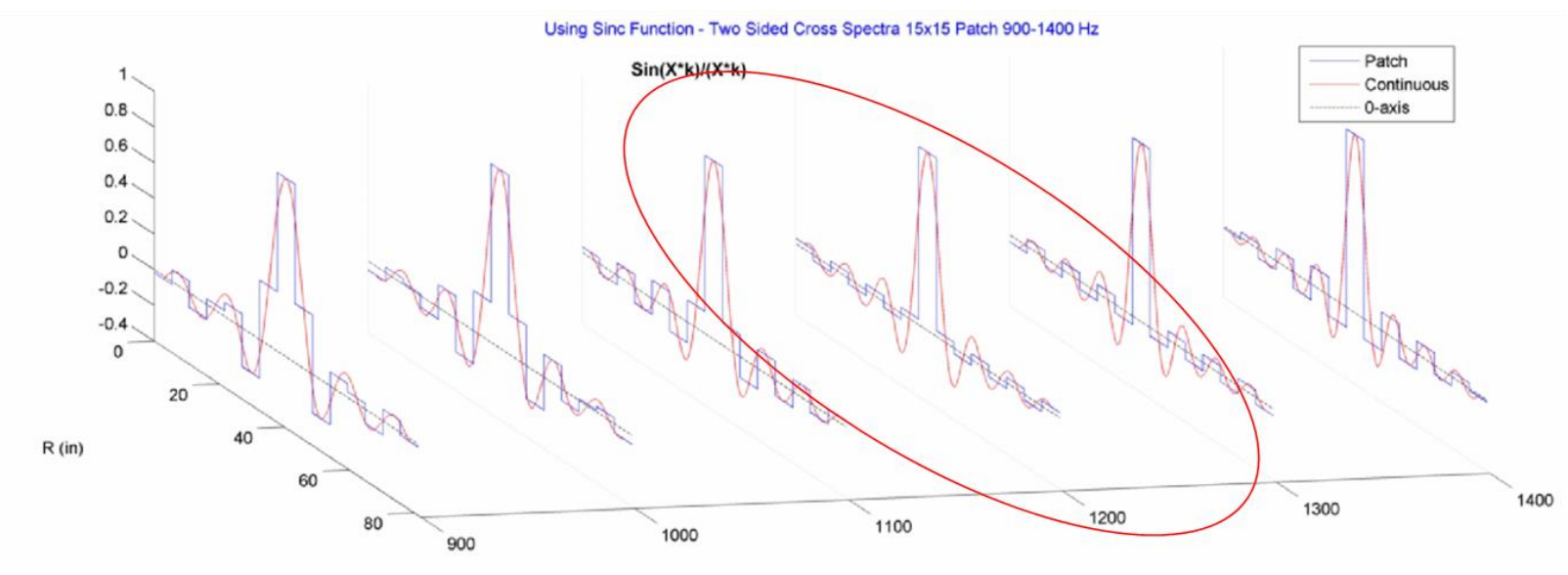

Figure 9. Spatial Correlation Function - The plot presents a single row of 15 patches from a $15 \times 15$ approximation of the Sinc spatial correlation function providing good approximations in the range from 900 to $1400 \mathrm{~Hz}$. Because the 5.4 in patch size is too coarse, it is approximately equal to $1 / 2$ the fluid wavelength in the range near $1200 \mathrm{~Hz}$. The result is that the continuous function details are not well approximated spatially, in fact the patch approximation magnitude approaches zero for all the side lobes of the function. A condition approximating the field similar to uncorrelated - "rain on the roof".
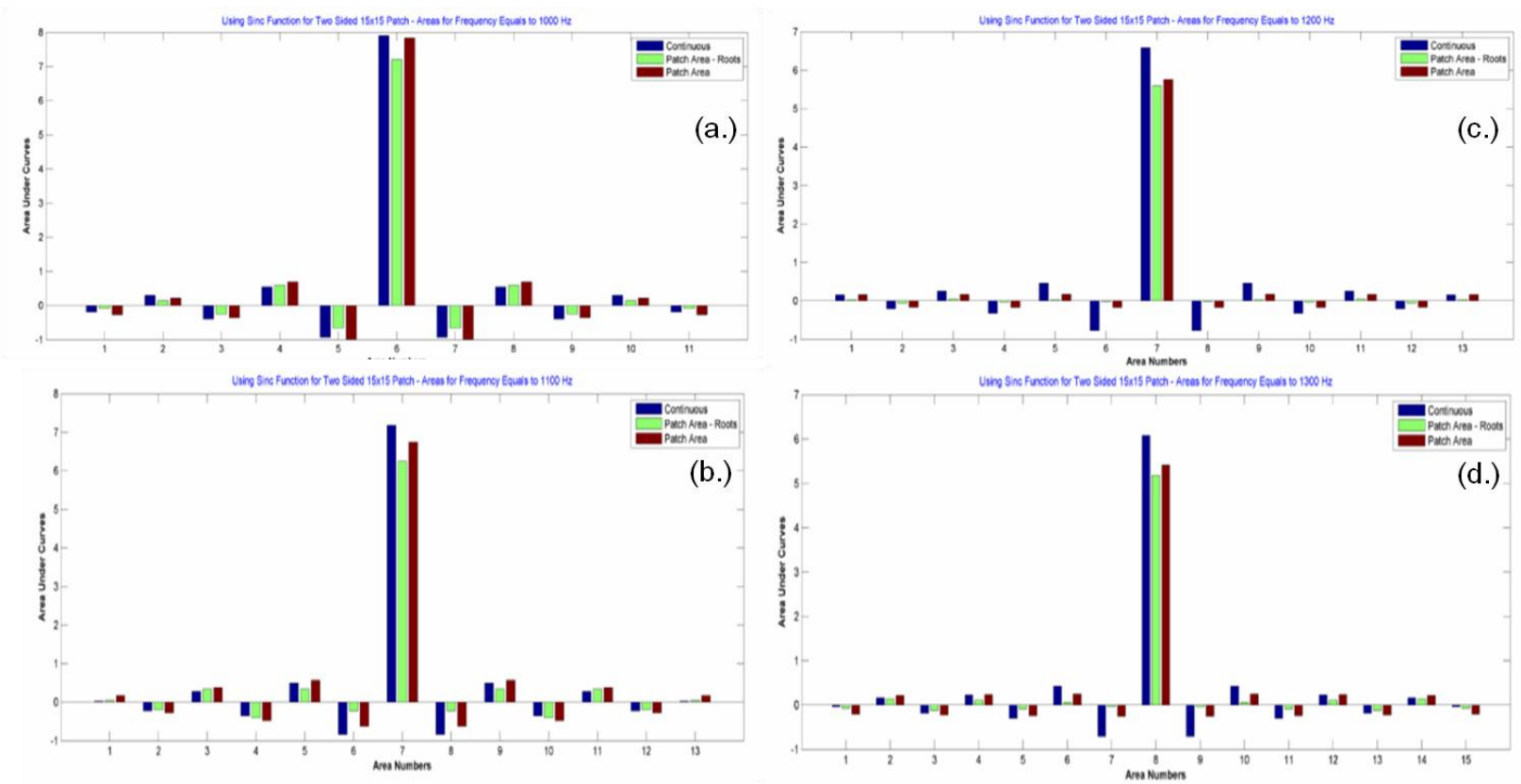

Figure 10. Spatial Correlation Function Area Comparisons: (a.) Correlation Area Comparison at 1000 Hz. (b.) Correlation Area Comparison at 1100 Hz. (c.) Correlation Area Comparison at 1200 Hz. (d.) Correlation Area Comparison at $1300 \mathrm{~Hz}$. 


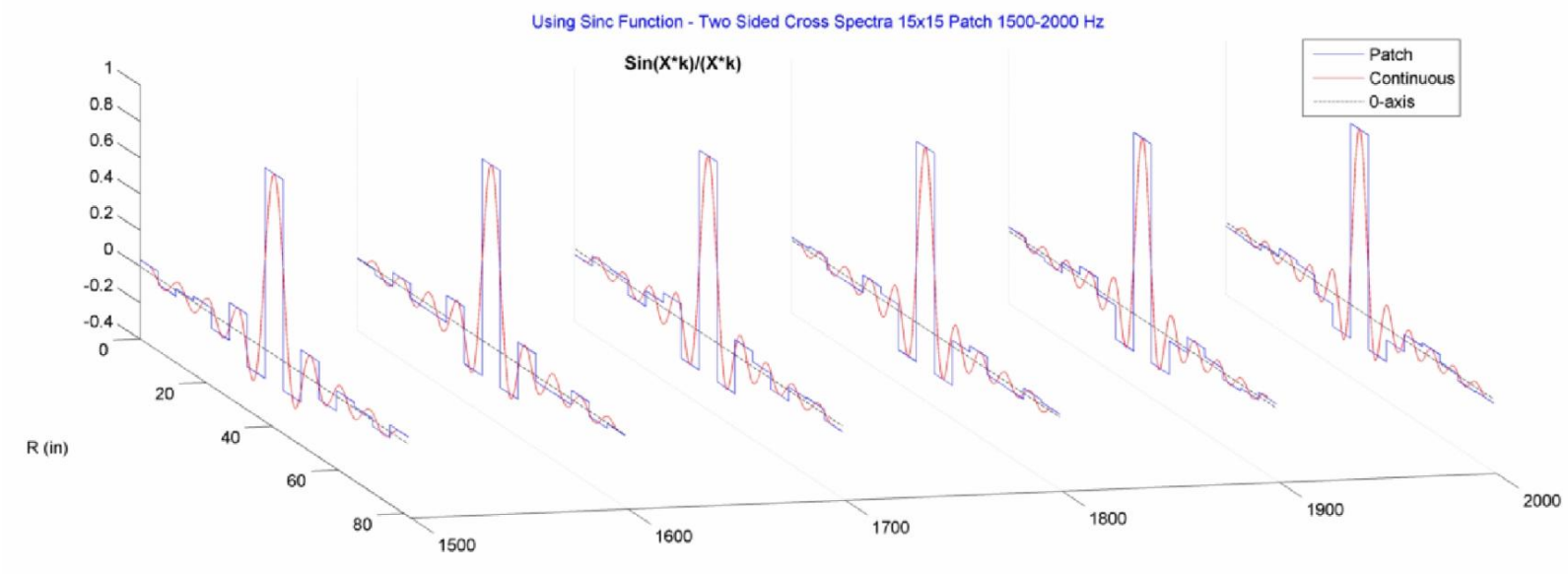

Figure 11. Spatial Correlation Function - The plot presents a single row of 15 patches from a $15 \times 15$ approximation of the Sinc spatial correlation function providing good approximations in the range from 1500 to $2000 \mathrm{~Hz}$.
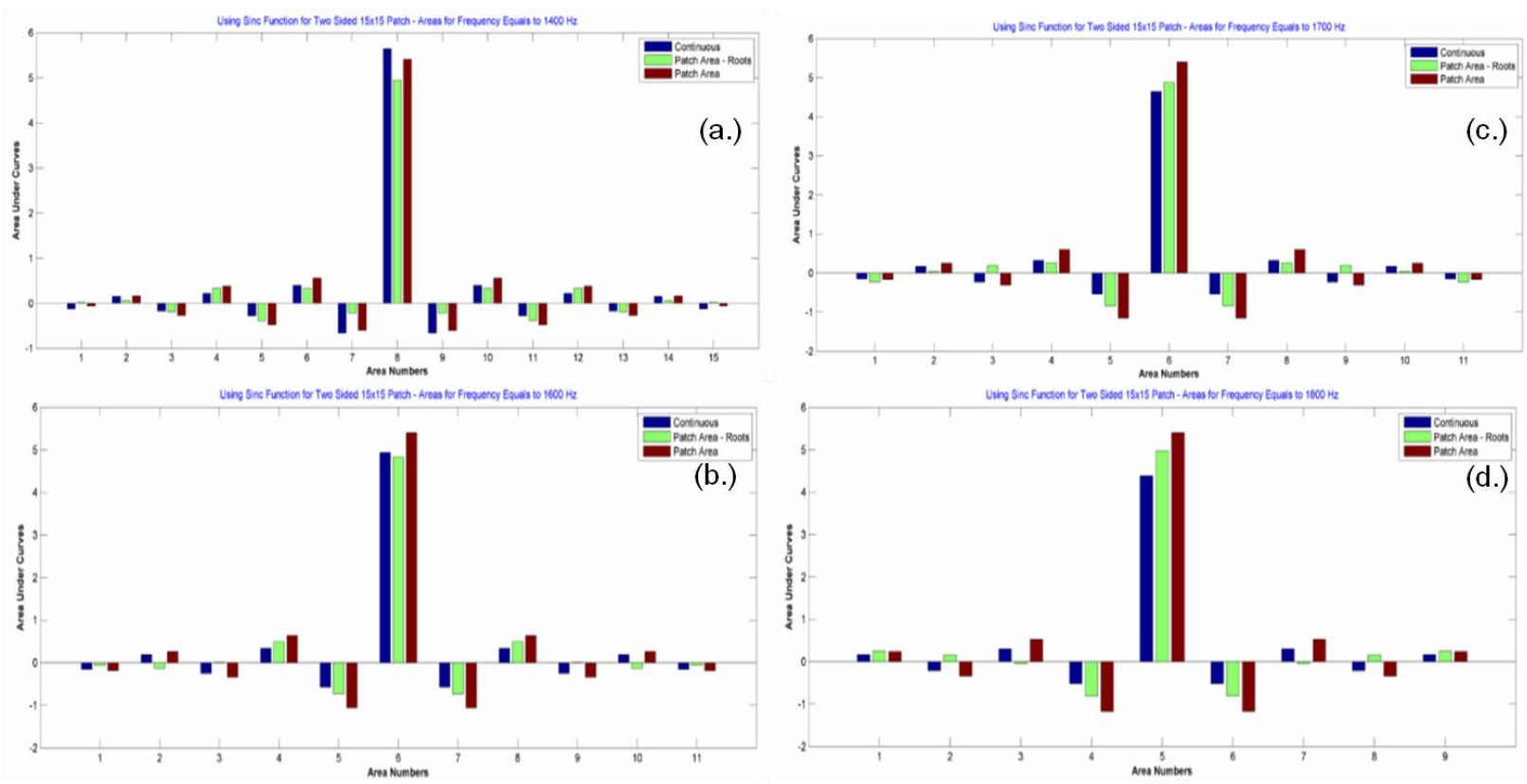

Figure 12. Spatial Correlation Function Area Comparisons: (a.) Correlation Area Comparison at 1400 Hz. (b.) Correlation Area Comparison at 1600 Hz. (c.) Correlation Area Comparison at 1700 Hz. (d.) Correlation Area Comparison at $1800 \mathrm{~Hz}$.

Figure 13 is provided so that the reader can appreciate an effect similar to Analog to digital aliasing of time data. When the patch assumption samples the intended correlation function too infrequently, a different wave form is represented by the patch approximation than that which was intended. 


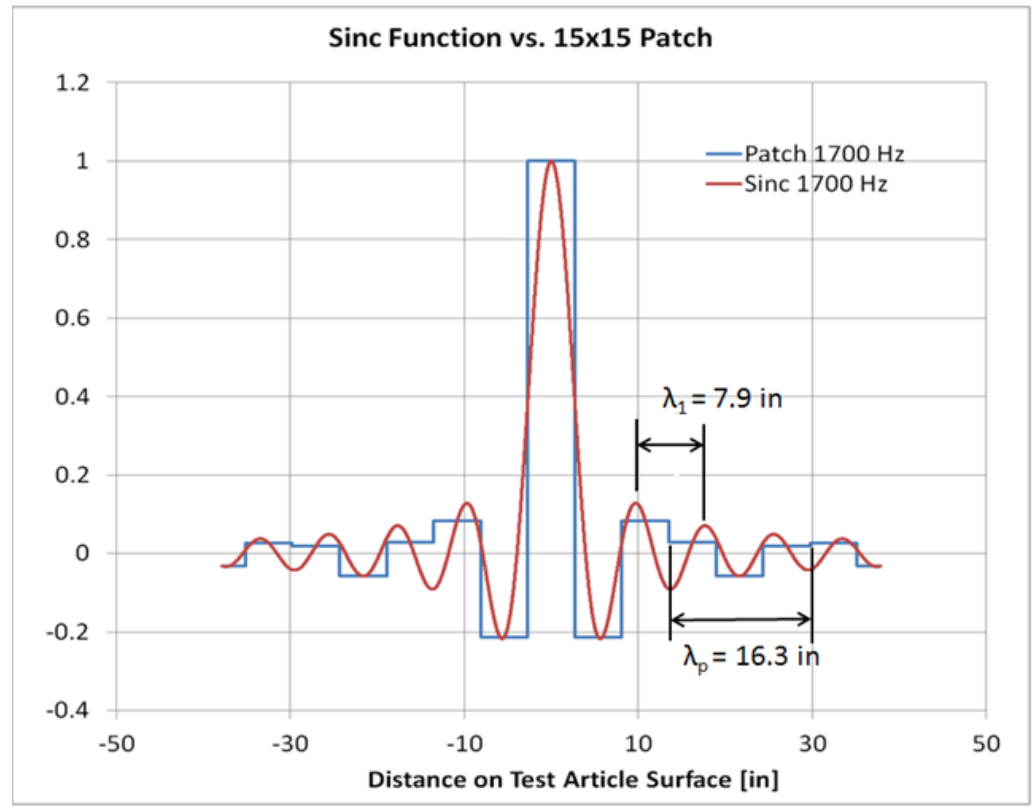

The continuous Sinc Function:

$$
\gamma=\frac{\sin (k x)}{k x}
$$

The apparent but not physical speed of sound implied by the poor patch approximation:

$$
\begin{aligned}
& c_{\text {apparent }}=f * \lambda_{p} \\
& c_{\text {apparent }}=1700 * 16.3 \\
& c_{\text {apparent }}=27710[\mathrm{in} / \mathrm{sec}]
\end{aligned}
$$

Figure 13. The details of how the continuous function varies spatially are not represented well at $1700 \mathrm{~Hz}$ because the patch size is too large to approximate the function well. The poor approximation resembles a frequency aliasing affect. This effect could produce a pseudo coincidence frequency for some designs.

\section{Vehicle Panel Response Convergence Study}

Actual vibration response was then calculated using the same vehicle panel FEM and several different assumptions for patch density. The applied pressure field was identical in each case except for the number of
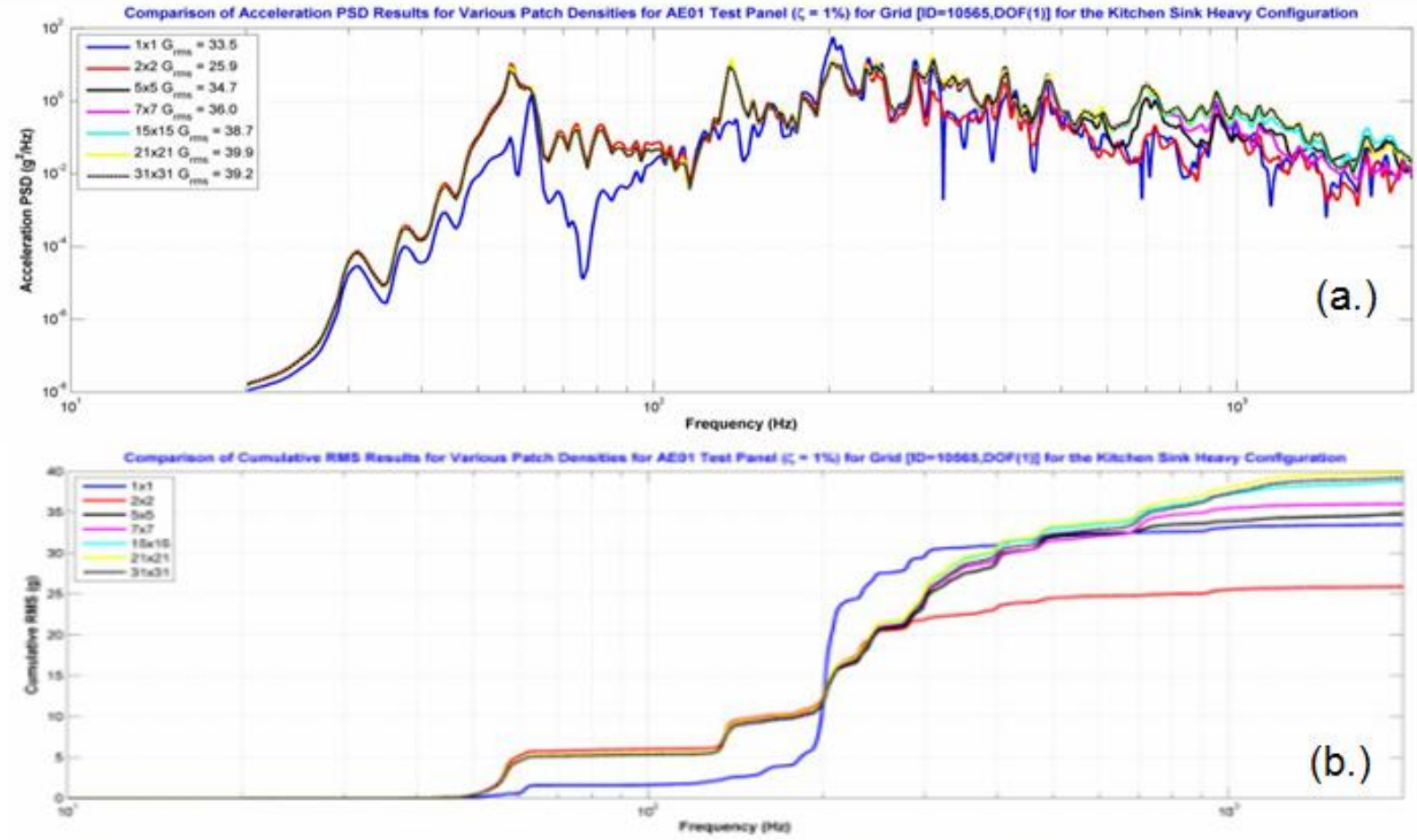

Figure 14. Convergence Study Results: (a.) Overlaid Response from 7 different patch density assumptions. (b.) Overlay of Cumulative RMS plots from the same 7 patch density assumptions. 
patches and therefore the fidelity of realized cross correlation relationships represented by the cross terms. The cases are summarized in Table 1. Of the three important structural modes in the range from $50-65 \mathrm{~Hz}$ (Figure 15) the totally correlated response case (the $1 \times 1$ blue response trace of Figure 14) is only effective at exciting the odd mode. It therefore provided a very poor approximation.
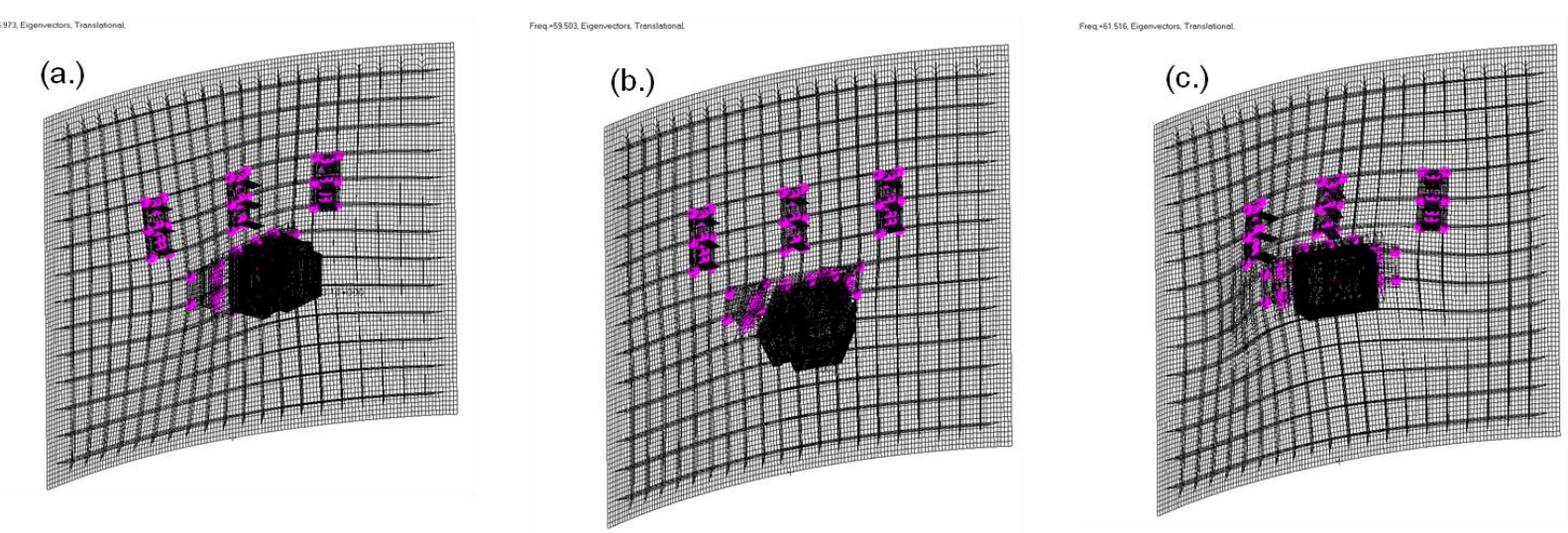

Figure 15. The first three system mode shapes of the test article (a.) $57.0 \mathrm{~Hz}$ an even mode with nodal line extending vertically near center of panel dividing two equal areas of panel which respond 180 degrees out of phase from each other (b.) $59.5 \mathrm{~Hz}$ another even mode with nodal line extending horizontally near center of panel dividing two nearly equal areas of panel which respond 180 degrees out of phase from each other (c.) $61.5 \mathrm{~Hz}$ an odd mode with the largest area of panel displacing in the same direction.

\section{Conclusion}

Spatial correlation function approximation using the patch method was illustrated across the frequency range for several different patch assumptions. Ranges where the approximation is not ideal were identified by qualitative/quantitative comparison. A convergence study illustrating the sensitivity to patch density was presented.

The recommend patch size should be based on the highest frequency range of interest. Maintain "Patch center to center distance" less than $1 / 2$ the fluid wavelength at highest frequency of interest. Note $1 / 3$ or $1 / 4$ of fluid wavelength is preferred.

A summary relating rectangular "Patch Size" to "Fluid Acoustic Wavelength" is provided in Table 1 below.

\begin{tabular}{|c|c|c|c|c|c|c|c|c|c|c|c|c|c|c|}
\hline \multirow{2}{*}{\multicolumn{4}{|c|}{\begin{tabular}{|l|} 
For a Vehicle Panel \\
of Length 81.125 in
\end{tabular}}} & \multirow{3}{*}{$\begin{array}{c}\text { Number of } \\
\text { Patches }\end{array}$} & \multirow{3}{*}{$\begin{array}{c}\text { Patch } \\
\text { Size [in] }\end{array}$} & \multicolumn{9}{|c|}{ Fraction of Wavelength } \\
\hline & & & & & & 67 in & 26.8 in & $19.1 \mathrm{in}$ & $14.9 \mathrm{in}$ & $12.2 \mathrm{in}$ & $10.3 \mathrm{in}$ & \multirow{2}{*}{$\begin{array}{l}8.9 \mathrm{in} \\
1500 \mathrm{~Hz}\end{array}$} & \multirow{2}{*}{$\begin{array}{l}6.4 \mathrm{in} \\
2100 \mathrm{~Hz}\end{array}$} & \multirow{2}{*}{$\begin{array}{l}4.3 \mathrm{in} \\
3100 \mathrm{~Hz}\end{array}$} \\
\hline Patc & Der & sty 7 & ials & & & $200 \mathrm{~Hz}$ & $500 \mathrm{~Hz}$ & $700 \mathrm{~Hz}$ & $900 \mathrm{~Hz}$ & $1100 \mathrm{~Hz}$ & $1300 \mathrm{~Hz}$ & & & \\
\hline 1 & $x$ & 1 & $=$ & 1 & 81.1 & 1.21 & 3.03 & 4.24 & 5.45 & 6.66 & 7.87 & 9.08 & 12.71 & 18.77 \\
\hline 2 & $x$ & 2 & $=$ & 4 & 40.6 & 0.61 & 1.51 & 2.12 & 2.72 & 3.33 & 3.94 & 4.54 & 6.36 & 9.38 \\
\hline 5 & $x$ & 5 & $=$ & 25 & 16.2 & 0.24 & 0.61 & 0.85 & 1.09 & 1.33 & 1.57 & 1.82 & 2.54 & 3.75 \\
\hline 7 & $x$ & 7 & $=$ & 49 & 11.6 & 0.17 & 0.43 & 0.61 & 0.78 & 0.95 & 1.12 & 1.30 & 1.82 & 2.68 \\
\hline 9 & $x$ & 9 & $=$ & 81 & 9.0 & 0.13 & 0.34 & 0.47 & 0.61 & 0.74 & 0.87 & 1.01 & 1.41 & 2.09 \\
\hline 11 & $x$ & 11 & $=$ & 121 & 7.4 & 0.11 & 0.28 & 0.39 & 0.50 & 0.61 & 0.72 & 0.83 & 1.16 & 1.71 \\
\hline 13 & $x$ & 13 & $=$ & 169 & 6.2 & 0.09 & 0.23 & 0.33 & 0.42 & 0.51 & 0.61 & 0.70 & 0.98 & 1.44 \\
\hline 15 & $x$ & 15 & $=$ & 225 & 5.4 & 0.08 & 0.20 & 0.28 & 0.36 & 0.44 & 0.52 & 0.61 & 0.85 & 1.25 \\
\hline 21 & $x$ & 21 & $=$ & 441 & 3.9 & 0.06 & 0.14 & 0.20 & 0.26 & 0.32 & 0.37 & 0.43 & 0.61 & 0.89 \\
\hline 31 & $x$ & 31 & $=$ & 961 & 2.6 & 0.04 & 0.10 & 0.14 & 0.18 & 0.21 & 0.25 & 0.29 & 0.41 & 0.61 \\
\hline
\end{tabular}

Table 1. Relating the patch density of example to the patch center to center distance. The yellow highlights correspond to patch assumption rows that are approximately $1 / 4$ of the fluid wavelength at each frequency listed in red above. The orange highlights correspond to patch assumption rows that are approximately $1 / 3$ of the fluid wavelength. 


\section{Acknowledgments}

The Authors would like thank and acknowledge Ms. Karen Oliver of MSFC/ EV31, as well as Ms. Lakiesha Hawkins of MSFC EV41 without whose support and vision the work could not have been accomplished.

\section{References}

${ }^{1}$ Peck, J., Smith, A., Fulcher, C.., LaVerde B., Hunt, R.., "Development of Component Interface Loads on a Cylindrical Orthogrid Vehicle Section from Test-Correlated Models of a Curved Panel," Proceedings of 2011 Spacecraft and Launch Vehicle Dynamic Environments Workshop, June 2011..

${ }^{2}$ Maasha, R., Towner, R., LaVerde B., Band, J., "Preliminary Correlation Results Summary for Mass Loaded Vehicle Panel Test Article Finite Element Models and Modal Survey TESSSA," Proceedings of 2011 Spacecraft and Launch Vehicle Dynamic Environments Workshop, June 2011.

${ }^{3}$ Rafaely, B., "Spatial-temporal correlation of a diffuse sound field," Institute of Sound and Vibration Research, University of Southampton, J. Acoust. Soc. Am. Vol. 107, No. 6, June 2000, pp. 3254-3258.

${ }^{4}$ Smith, A., Davis, R. B., LaVerde, B., Jones, D., "Reporting Recommended Patch Density from Vehicle Panel Vibration Convergence Studies using both DAF and TBL Fits of the Spatial Correlation Function," Spacecraft and Launch Vehicle Dynamics Environments Workshop, June, 2012. 